\title{
A GLASER TWIST: FOCUS ON THE MIXTURE PARAMETERS
}

\author{
HENRY W. BLOCK, ${ }^{* * *}$ University of Pittsburgh \\ NAFTALI A. LANGBERG, ${ }^{* * *}$ University of Haifa \\ THOMAS H. SAVITS, ${ }^{*}$ University of Pittsburgh
}

\begin{abstract}
In this paper we introduce a variation on Glaser's method for determining the shape of the failure rate function of a mixture. It has often been seen that the shape of the failure rate depends on the mixing parameter $q$. Our method provides an explanation for this phenomenon. We then illustrate our technique with the mixture of an exponential and a gamma density for all possible cases.
\end{abstract}

Keywords: Reliability; failure rate function; bathtub failure rate

2010 Mathematics Subject Classification: Primary 62N05

\section{Introduction}

For the past several years, there has been a great deal of interest in determining the shape of the failure rate function of mixtures. Mixtures are important in reliability theory since it is widely believed that most populations are comprised of hetereogeneous subpopulations. See, e.g. Block et al. (2003a) for a more detailed discussion. For extensive discussions concerning mixtures of survival functions, see the recent books of Lai and Xie (2006) and Marshall and Olkin (2007).

In Glaser's (1980) seminal paper, he introduced an indirect method for studying the failure rate. Instead of directly investigating the failure rate function $r(t)=f(t) / \bar{F}(t)$, he considered the shape of a related function $\eta(t)=-\mathrm{d} \log f(t) / \mathrm{d} t=-f^{\prime}(t) / f(t)$. Generally, this is a much simpler object since it does not involve the survival function $\bar{F}(t)$. In that paper, he showed, for example, that if $\eta(t)$ is increasing then $r(t)$ is also increasing, and if $\eta(t)$ has a bathtub shape then $r(t)$ has either a bathtub shape or is increasing. This method has since been extended to functions having more than one change of monotonicity in Gupta and Warren (2001) and Savits (2003).

In this paper we introduce a slightly different approach, although it is still based on Glaser's (1980) result. Consider a mixture $f(t)=p g(t)+q h(t)$. Instead of concentrating on the above function $\eta(t)$ as a function of $t$ only, we will show that $\eta^{\prime}(t)$ is a quadratic function of $q$ when we make use of the relation $p=1-q$. This allows us to study the shape characteristics of $\eta(t)$ as a function of the roots, $Q_{1}(t)$ and $Q_{2}(t)$, of the quadratic equation for $0 \leq Q_{1}(t), Q_{2}(t) \leq 1$, and, hence, to make direct inferences about the shape of the failure rate function $r(t)$ and its dependence on the mixing parameter $q$.

Received 9 August 2011; revision received 6 June 2012.

* Postal address: Department of Statistics, University of Pittsburgh, 2717 Cathedral of Learning, Pittsburgh, PA 15260, USA.

Supported by NSA Grant H 98230-07-1-0018.

** Email address: hwb@pitt.edu

*** Postal address: Department of Statistics, Haifa University, Mount Carmel, Haifa 31999, Israel. 
Our method is detailed in Section 1. In Section 2 we apply our technique to a mixture of one exponential and one gamma density. One case, in particular, explicitly demonstrates how the mixture failure rate shape depends on the mixing parameter $q$. In this case, case (b), it is shown that, for a certain set of parameter values of the exponential and gamma densities, an explicit value $0<q^{*}<1$ can be calculated such that, for $1>q \geq q^{*}$, the failure rate of the mixture is increasing, while, for $0<q<q^{*}$, the failure rate has either a modified bathtub shape or is increasing.

A few examples of these types of result were given in Block et al. (2003a). These authors considered two different mixtures of an exponential and a simple gamma with shape parameter 2, and showed that several differently shaped failure rates are possible. In Example 2.3 of Block et al. (2003a), for an exponential with scale parameter $\lambda=1$, which is smaller than the scale parameter $\lambda_{0}=4$ of the gamma, the failure rate has an upside-down shape. If, on the other hand, the exponential has scale parameter $\lambda=4$, which is larger than the scale parameter $\lambda_{0}=1$ of the gamma, the resulting failure rate is bathtub shaped. Whether these results generalized was not known.

In this paper, as the application of our method, we classify all of the shapes obtained by mixing an exponential with scale parameter $\lambda$ and a gamma with scale parameter $\lambda_{0}$ and shape parameter $\phi$. Glaser (1980) obtained results when the scale parameters are equal.

Before we close this section, we need to introduce some terminology, not all of which is standard in the reliability literature. In this paper, increasing will mean nondecreasing and decreasing will mean nonincreasing. A life distribution whose failure rate is increasing (decreasing) but not constant is said to be IFR (DFR). If it has exactly one definite change of monotonicity from decreasing to increasing (from increasing to decreasing) it is called bathtub or BT for short (upside-down bathtub or UB for short). Lastly, if the failure rate has exactly two definite changes of monotonicity from increasing to decreasing to increasing (decreasing to increasing to decreasing), it is said to have a modified bathtub shape or MBT (modified upside-down bathtub shape or MUB). By convention, an exponential distribution is included in both the IFR and DFR classes since its failure rate is constant.

\section{General method}

Consider a mixture of two subpopulations

$$
f(t)=p g(t)+q h(t),
$$

where $0<p<1$ and $p+q=1$. According to Glaser (1980), the failure rate of the mixture can be determined from the shape characteristics of

$$
\eta(t)=-\frac{\mathrm{d}}{\mathrm{d} t} \log f(t)=-\frac{f^{\prime}(t)}{f(t)} .
$$

Its shape can be determined by investigating the monotonicity properties of $\eta(t)$ or, equivalently, by the sign behavior of its derivative $\eta^{\prime}(t)$. Assuming that the densities $g(t)$ and $h(t)$ are positive on $(0, \infty)$ and sufficiently smooth, we introduce the function

$$
K(t)=\eta^{\prime}(t) f^{2}(t)=A_{11}(t) p^{2}+A_{12}(t) p q+A_{22}(t) q^{2},
$$

with

$$
\begin{gathered}
A_{11}(t)=\left[g^{\prime}(t)\right]^{2}-g(t) g^{\prime \prime}(t), \quad A_{22}(t)=\left[h^{\prime}(t)\right]^{2}-h(t) h^{\prime \prime}(t), \\
\text { and } A_{12}(t)=2 g^{\prime}(t) h^{\prime}(t)-g(t) h^{\prime \prime}(t)-g^{\prime \prime}(t) h(t) .
\end{gathered}
$$

We note that $K(t)$ has the same sign properties as $\eta^{\prime}(t)$. 
Glaser (1980) illustrated his method by directly examining $\eta^{\prime}(t)$ for various standard densities $(q=0)$ as well as for mixture models $(0<q<1)$. In particular, he considered the case of a mixture of two gamma densities having the same scale parameter. Glaser was able to classify the failure rate shape for all but one particular case of the shape parameters. In an attempt to settle the final case, Gupta and Warren (2001) extended Glaser's technique. They then applied it to the remaining undetermined case with partial success: they were able to show graphically that the shape depended on the mixing parameter $q$.

In several later papers, Block et al. (2008) considered a mixture model in which $g(t)$ is itself a continuous mixture of exponentials, i.e.

$$
g(t)=\int_{(0, \infty)} \lambda \mathrm{e}^{-\lambda t} P(\mathrm{~d} \lambda)=\mathrm{E}\left[\Lambda \mathrm{e}^{-\Lambda t}\right],
$$

and $h(t)$ is a single IFR gamma with shape parameter $\phi>2$ and scale parameter $\lambda_{0}>0$. In this case the failure rate of the mixture is BT, possibly degenerate, when the support of $P$ is a subset of $\left(\lambda_{0}, \infty\right)$. The above result was extended in Block et al. (2010) to the case where now $h(t)$ is also a mixture of IFR gammas having the same scale parameter $\lambda_{0}$ :

$$
h(t)=\mathrm{E}\left[\frac{\mathrm{e}^{-\lambda_{0} t} \lambda_{0}^{\Phi} t^{\Phi-1}}{\Gamma(\Phi)}\right] .
$$

For a certain set of parameter values, they again concluded that the failure rate of the mixture is BT. For another set of parameters, Block et al. (2012) showed that the above continuous mixture model of gammas and exponentials yielded a UB-shaped (possibly degenerate) failure rate.

In each of the three articles discussed above, the terms $A_{11}(t), A_{22}(t)$, and $A_{12}(t)$ of $K(t)$ in (2.1) were separately analyzed. No information about the mixing parameters $p$ and $q$ were used except that they were positive. The information that they were tied together through the equation $p+q=1$ was ignored. Our new variation makes use of this natural restriction.

To take advantage of this constraint, we substitute $p=1-q$ and rewrite $K(t)$ as

$$
K(t ; q)=B_{2}(t) q^{2}+B_{1}(t) q+B_{0}(t)
$$

where

$$
\begin{gathered}
B_{0}(t)=A_{11}(t), \quad B_{1}(t)=A_{12}(t)-2 A_{11}(t), \\
\text { and } \quad B_{2}(t)=A_{11}(t)+A_{22}(t)-A_{12}(t) .
\end{gathered}
$$

We now observe that $K(t ; q)$ is a quadratic in the parameter $0<q<1$ and, hence, has two roots, denoted by $Q_{1}(t)$ and $Q_{2}(t)$. We propose to study these roots. Since

$$
K(t ; q)=B_{2}(t)\left[q-Q_{1}(t)\right]\left[q-Q_{2}(t)\right],
$$

the sign properties of $K(t ; q)$ and, hence, $\eta^{\prime}(t)$ can be inferred from the behavior of $B_{2}(t)$ and the roots $Q_{1}(t)$ and $Q_{2}(t)$.

We illustrate the results of our technique in the next section where we consider a mixture model of one exponential and one gamma density. The details are included in Appendix A. We have also successfully applied the technique to the mixture model of two gammas having the same shape parameter as well as to the previously studied model (e.g. Glaser (1980)) of two gammas having the same scale parameter, but these will be left for a possible future paper. 


\section{Application of the Glaser twist method}

We apply the Gleser twist method to determine the shape of all possible mixtures of an exponential and a gamma distribution.

Consider the mixture model of the form

$$
f(t)=p g(t)+q h(t), \quad 0<p, q<1, p+q=1,
$$

with

$$
g(t)=\lambda \mathrm{e}^{-\lambda t} \quad \text { and } \quad h(t)=\frac{\lambda_{0}\left(\lambda_{0} t\right)^{\phi-1} \mathrm{e}^{-\lambda_{0} t}}{\Gamma(\phi)} .
$$

Without loss of generality, we may take $\lambda_{0}=1$.

The specific failure rate behaviors can be obtained for the above mixture using the method of Section 1. These are presented for all possible cases, given as cases (a) through (g) (with several subcases) in Table 1 . We introduce the parameter $v$, where $v=1 / q$. The only remaining quantity which we have not defined is $v^{*}$, which is defined in the proof of (b) in Appendix A.

Notes. Some of the above results were obtained by others. Case (a) is a special case considered in Block et al. (2008) and case (c) was also considered but not completely delineated in the same publication. Cases (e) and (f) are considered in Block et al. (2012). Finally, case (g) was treated in Glaser (1980).

We begin our analysis of the mixture as follows. From (2.2), it is easy to check that

$$
A_{11}(t)=0, \quad A_{22}(t)=\frac{\phi-1}{\Gamma^{2}(\phi)} t^{2(\phi-2)} \mathrm{e}^{-2 t}, \quad \text { and } \quad A_{12}(t)=\frac{\lambda \mathrm{e}^{-t(\lambda+1)}}{\Gamma(\phi)} t^{\phi-3} P(t),
$$

with

$$
P(t)=(\phi-1)(2-\phi)+2(\phi-1)(1-\lambda) t-(1-\lambda)^{2} t^{2} .
$$

Thus, from (2.1) we obtain

$$
K(t)=\left[\frac{\lambda \mathrm{e}^{-t(\lambda+1)}}{\Gamma(\phi)} t^{\phi-3} P(t)\right] p q+\left[\frac{\phi-1}{\Gamma^{2}(\phi)} t^{2(\phi-2)} \mathrm{e}^{-2 t}\right] q^{2} .
$$

TABLe 1.

\begin{tabular}{ccccc}
\hline Case & Criterion & Subcase & Criterion & Behavior \\
\hline (a) & $\lambda>1, \phi>2$ & & & BT \\
(b) & $\lambda>1,1<\phi<2$ & (b.1) & $1<v \leq v^{*}$ & IFR \\
& & (b.2) & $v^{*}<v, p \lambda \geq 1$ & MBT \\
& & (b.3) & $v^{*}<v, p \lambda<1$ & IFR or MBT \\
(c) & $\lambda>1, \phi=2$ & (c. 1$)$ & $1<v \leq 1+[2 \lambda(\lambda-1)]^{-1}$ & IFR \\
& & $($ c. 2$)$ & $1+[2 \lambda(\lambda-1)]^{-1}<v, \lambda^{2} p \leq 1$ & IFR \\
& & $($ c. 3$)$ & $1+[2 \lambda(\lambda-1)]^{-1}<v, \lambda^{2} p>1$ & BT \\
(d) & $0<\lambda<1, \phi>2$ & & & MUB \\
(e) & $0<\lambda<1,1<\phi<2$ & & & UB \\
(f) & $0<\lambda<1, \phi=2$ & & & UB \\
(g) & $\lambda=1,1<\phi \leq 2$ & & & IFR \\
& $\lambda=1, \phi>2$ & & BT \\
\hline
\end{tabular}


Since the sign properties of $K(t)$ are unaffected by multiplying with a positive function, we may renormalize $K(t)$ as

$$
\tilde{K}(t)=\Gamma^{2}(\phi) \mathrm{e}^{2 t} t^{3-\phi} K(t)=\left[\lambda \Gamma(\phi) \mathrm{e}^{t(1-\lambda)} P(t)\right] p q+\left[(\phi-1) t^{\phi-1}\right] q^{2} .
$$

Expressions (2.3) and (2.4) now become

$$
\tilde{K}(t ; q)=\tilde{B}_{2}(t) q^{2}+\tilde{B}_{1}(t) q=q \tilde{B}_{2}(t)[q-\tilde{Q}(t)]=q \tilde{B}_{2}(t)[q-Q(t)],
$$

where

$$
\begin{gathered}
\tilde{B}_{1}(t)=\lambda \Gamma(\phi) \mathrm{e}^{t(1-\lambda)} P(t), \quad \tilde{B}_{2}(t)=(\phi-1) t^{\phi-1}-\lambda \Gamma(\phi) \mathrm{e}^{t(1-\lambda)} P(t) \\
\tilde{Q}(t)=-\frac{\tilde{B}_{1}(t)}{\tilde{B}_{2}(t)}, \quad Q(t)=-\frac{B_{1}(t)}{B_{2}(t)},
\end{gathered}
$$

and the last line of (3.2) follows since

$$
\frac{\tilde{B}_{1}(t)}{\tilde{B}_{2}(t)}=\frac{B_{1}(t)}{B_{2}(t)}
$$

if the denominators are assumed to be nonzero. Note that in this case, for $(2.5), Q_{1}(t)=0$, while we denote $Q_{2}(t)$ by $Q(t)$ for simplicity.

In the remainder of the paper we analyze all possible behaviors of the mixture failure rate for all $\lambda>0$ and $\phi>0$. Since the gamma density has a decreasing failure rate when $0<\phi \leq 1$, it follows from the closure result of Esary et al. (1970) that the mixture failure rate is also decreasing for all $\lambda>0$. Hence, we need only consider the case when $\phi>1$. Before delineating the various cases, however, we will first state the following useful facts (see Block et al. (2003a)).

Proposition 3.1. For mixture (3.1) with $\lambda_{0}=1, \phi>1$, and $\lambda>0$,

$$
f(t)=p \lambda \mathrm{e}^{-\lambda t}+q \frac{t^{\phi-1} \mathrm{e}^{-t}}{\Gamma(\phi)} .
$$

The mixture failure rate $r(t)$ satisfies

$$
\begin{aligned}
r(0+) & =p \lambda, \\
r^{\prime}(0+) & = \begin{cases}+\infty & \text { if } 1<\phi<2, \\
q\left(1-p \lambda^{2}\right) & \text { if } \phi=2, \\
-p q \lambda^{2} & \text { if } \phi>2,\end{cases} \\
r(+\infty) & =\min (\lambda, 1),
\end{aligned}
$$

and

$$
r(t) \text { is } \begin{cases}\text { ultimately strictly decreasing } & \text { if } 0<\lambda<1, \\ \text { ultimately strictly increasing } & \text { if } \lambda>1 .\end{cases}
$$

Proof. The calculation of $r(0+)$ is straightforward. The expression for $r^{\prime}(0+)$ follows from Remark 2.4 of Block et al. (2003a). The quantity $r(+\infty)$ follows from Theorem 2.1 of the same paper. Now let $r_{1}(t)=\lambda$ be the failure rate of the exponential (with survival function $\left.\bar{F}_{1}(t)=\exp (-\lambda t)\right)$, and let $r_{2}(t)$ be the failure rate of the gamma with scale parameter 1 and 
shape parameter $\phi$ (with survival function $\bar{F}_{2}(t)$ ). For $\lambda>1$, using the facts that $r_{2}(t) \sim$ $1+(1-\phi) / t$ and $r_{2}^{\prime}(t) \sim(\phi-1) / t^{2}$ as $t \rightarrow \infty$, Theorem 2.4 of Block et al. (2003a) can be applied. This reveals that $r(t)$ is ultimately strictly increasing.

The corresponding result for $0<\lambda<1$ takes some more work. First, either directly or using the proof of Theorem 2.4 of Block et al. (2003a), we obtain

$$
\begin{aligned}
r^{\prime}(t) & =q r_{2}^{\prime}(t) \frac{\bar{F}_{2}(t)}{\bar{F}(t)}-p q\left[\lambda-r_{2}(t)\right]^{2} \frac{\bar{F}_{1}(t)}{\bar{F}(t)} \frac{\bar{F}_{2}(t)}{\bar{F}(t)} \\
& =q \frac{\bar{F}_{2}(t)}{\bar{F}(t)}\left\{r_{2}^{\prime}(t)-\left[\lambda-r_{2}(t)\right]^{2} p \frac{\bar{F}_{1}(t)}{\bar{F}(t)}\right\} .
\end{aligned}
$$

Applying Lemma 2.1 of Block et al. (2003a), we find that $p \bar{F}_{1}(t) / \bar{F}(t)$ goes to 1 as $t$ goes to $\infty$. Since $r_{2}(t) \rightarrow 1$ and $r_{2}^{\prime}(t) \rightarrow 0$ as $t \rightarrow \infty$, it follows that $r^{\prime}(t)$ is eventually negative and so, for $0<\lambda<1, r(t)$ is eventually strictly decreasing.

The following proposition is a summary of results from Savits (2003) and is used in most of the cases which follow. In general, these results state that if $\eta$ has a certain number, $k$, of changes of monotonicity then the number of changes of monotonicity of the failure rate is no more than $k$.

Proposition 3.2. Consider a distribution with a positive density function $f$ on $(0, \infty)$. Assume that $f$ is continuously differentiable there, and set $\eta(t)=-f^{\prime}(t) / f(t)$. Then the following statements hold.

(a) If $\eta$ is increasing then the distribution is IFR and if $\eta$ is decreasing then the distribution is DFR.

(b) If $\eta^{\prime}$ has a sign change from - to $+($ from + to -$)$ then the distribution is either BT or IFR (either UB or DFR).

(c) If $\eta$ has two sign changes from + to - to $+(-$ to + to -$)$ then the distribution is one of IFR, $B T$, or MBT (one of DFR, $U B$, or MUB).

Proof. (a) This follows from Corollary 3.1(i) of Savits (2003).

(b) This follows from Corollary 3.1(iii) and (iv) of Savits (2003).

(c) This follows from Theorem 3.1 of Savits (2003) in the same way that (b) follows from the same result.

Remark 3.1. Proposition 3.2(a) and (b) were first obtained in Glaser (1980).

Using Proposition 3.2, it can now be shown that the failure rate of the mixture of one exponential and one gamma distribution must be one of the following: IFR, DFR, BT, UB, MBT, or MUB. The results are summarized in Table 1 at the beginning of this section. The details are given in Appendix A.

\section{Review of previous literature, discussions, and concluding remarks}

The failure rate of a mixture is a rather intricate object. Many examples of a mixture of even two standard subpopulations can have a failure rate with a surprising number of possible shapes. Several examples are delineated in Block et al. (2003a). One of the few early successes in this area was the famous result of Esary et al. (1970) which states that the failure rate of an arbitrary mixture of decreasing failure rate distributions is again decreasing. Related but 
different results were obtained in Kemperman (1991), who studied the shape of the density of mixtures. Except for a result due to Glaser (1980) little was known about the 'global' shape of the failure rate for mixtures.

Instead of attempting to describe the entire shape of the failure rate of mixtures, some authors limited their investigations to describing its initial and final behaviors. Although the 'middle' life of the failure rate has generally not been determined, these results have been useful in providing some insight into the behavior of mixtures. A first result in this direction was due to Block et al. (1993). They showed the intuitive result that the asymptotic failure rate of a mixture tends to the asymptotic failure rate of the strongest subpopulation. Block and Joe (1997) considered the asymptotic monotonicity of the mixture failure rate. Their result was later extended and refined in Block et al. (2003a). These latter authors also investigated the initial behavior of the failure rate. The major result of these investigations was that in general the failure rate of the mixture has the same eventual monotonicity as the failure rate of its strongest component.

As mentioned earlier, the failure rate of a mixture is an intricate function of the individual failure rates of its subpopulations. Thus, a direct analysis of its failure rate is often a difficult task. One notable success with a direct calculation was in Block et al. (2003b) for the case of two subpopulations, each having an increasing linear failure rate. In this paper, among other things, these authors were also able to show that the shape of the failure rate can depend on the mixing parameter.

\section{Appendix A}

Case $(a): \lambda>1$ and $\phi>2$. We first consider the roots $s_{1}$ and $s_{2}$ of the quadratic expression $P(t)=0$ :

$$
s_{1}=\frac{\phi-1+\sqrt{\phi-1}}{1-\lambda}, \quad s_{2}=\frac{\phi-1-\sqrt{\phi-1}}{1-\lambda} .
$$

Thus, $s_{1}<s_{2}<0$. Consequently, $P(t)<0$ and, hence, $\tilde{B}_{1}(t)<0$ for all $t>0$ since $P(0)=(\phi-1)(2-\phi)<0$. Clearly, then $\tilde{B}_{2}(t)>0$ for all $t>0$.

We next investigate $Q(t)$. It is not hard to show that $Q(0+)=1$ while $Q(+\infty)=0$. To determine the monotonicity of $Q(t)$, we compute its derivative:

$$
Q^{\prime}(t)=\frac{\lambda(\phi-1) \Gamma(\phi) \mathrm{e}^{t(1-\lambda)} t^{\phi-2} R(t)}{\left[\tilde{B}_{2}(t)\right]^{2}} .
$$

Here

$$
R(t)=(2-\phi)(\phi-1)^{2}+3(1-\lambda)(\phi-1)(\phi-2) t+(1-\lambda)^{2}(5-3 \phi) t^{2}+(1-\lambda)^{3} t^{3} .
$$

The cubic equation $R(t)$ has three roots given by

$$
t_{1}=\frac{\phi-1}{1-\lambda}, \quad t_{2}=\frac{\phi-2+\sqrt{2-\phi}}{1-\lambda}, \quad \text { and } \quad t_{3}=\frac{\phi-2-\sqrt{2-\phi}}{1-\lambda} .
$$

Since $\phi>2, t_{2}$ and $t_{3}$ are complex while $t_{1}$ is real and negative. Since $R(0+)=$ $(2-\phi)(\phi-1)^{2}<0, R(t)<0$ for all $t>0$. Thus, $Q(t)$ is a strictly decreasing function of $t$. It thus follows from (3.2) that, given any $0<q<1$,

$$
\tilde{K}(t ; q) \begin{cases}<0 & \text { for } 0<t<\xi \\ >0 & \text { for } \xi<t\end{cases}
$$


where

$$
\xi=\inf \{t>0: Q(t)<q\} .
$$

Since $\eta^{\prime}(t)$ has only one sign change which goes from - to + , it follows from the results of Proposition 3.2 that the mixture failure rate $r(t)$ is either BT or IFR. But, from Proposition 3.1, we know that $r^{\prime}(0+)=-\lambda^{2} p q<0$ and, hence, cannot be IFR. Thus, $r(t)$ must be BT.

Case $(b): \lambda>1$ and $1<\phi<2$. In the remaining cases, a complication arises in the analysis of the term $\tilde{B}_{2}(t)$. It turns out to be more advantageous to rewrite $\tilde{K}(t ; q)$ in $(3.2)$ as

$$
L(t ; v)=v^{2} \tilde{K}\left(t ; \frac{1}{v}\right)=\tilde{B}_{1}(t) v+\tilde{B}_{2}(t)=\tilde{B}_{1}(t)[v-V(t)],
$$

with $v>1$ and

$$
V(t)=-\frac{1}{Q(t)}=-\frac{\tilde{B}_{2}(t)}{\tilde{B}_{1}(t)}
$$

if $\tilde{B}_{1}(t) \neq 0$. The advantage of (A.2) over (3.2) is that we can more easily determine the behavior of $\tilde{B}_{1}(t)$ since it is a quadratic function of $t$ with known roots $s_{1}$ and $s_{2}$ given in (A.1). Also,

$$
V^{\prime}(t)=-\frac{Q^{\prime}(t)}{Q^{2}(t)} \quad \text { or } \quad\left[\tilde{B}_{1}(t)\right]^{2} V^{\prime}(t)=-\lambda(\phi-1) \Gamma(\phi) \mathrm{e}^{t(1-\lambda)} t^{\phi-2} R(t) .
$$

Here we now have $s_{1}<t_{1}<t_{2}<0<s_{2}<t_{3}$ and so

$$
\tilde{B}_{1}(t) \begin{cases}>0 & \text { if } 0<t<s_{2} \\ <0 & \text { if } s_{2}<t\end{cases}
$$

noting that $\tilde{B}_{1}(0)=\lambda \Gamma(\phi)(\phi-1)(2-\phi)>0$, and

$$
R(t) \begin{cases}>0 & \text { if } 0<t<t_{3} \\ <0 & \text { if } t_{3}<t\end{cases}
$$

Consequently, $V(t)$ is strictly decreasing on $\left(0, s_{2}\right)$, strictly decreasing on $\left(s_{2}, t_{3}\right)$, and strictly increasing on $\left(t_{3}, \infty\right)$. Since $V(0+)=1$ and $\tilde{B}_{1}\left(s_{2}\right)=0$, it must be that $V\left(s_{2}-\right)=-\infty$ while $V\left(s_{2}+\right)=+\infty$. We also know that $V(+\infty)=+\infty$ and, thus, $V(t)$ achieves its minimum on $\left(s_{2}, \infty\right)$ at $t=t_{3}$. If we evaluate $V(t)$ at $t=t_{3}$, we obtain

$$
V\left(t_{3}\right)=1-\frac{(\phi-1) t_{3}^{\phi-1}}{\lambda \Gamma(\phi) \mathrm{e}^{t_{3}(1-\lambda)} P\left(t_{3}\right)} .
$$

Since $P\left(t_{3}\right)<0$, it follows that $V\left(t_{3}\right)>1$. Set $v^{*}=V\left(t_{3}\right)$, and let $q^{*}=1 / v^{*}$. For fixed $v>1$, we have several subcases to consider.

Subcase (b.1): $1<v \leq v^{*}$. It can be seen from (A.2) and (A.3) that $L(t ; v) \geq 0$ with equality only if $t=t_{3}$ and $v=v^{*}$ for all $t>0$. Thus, $\eta(t)$ is strictly increasing and so $r(t)$ must be IFR.

Remark A.1. Although $V(t)$ is discontinuous wherever $\tilde{B}_{1}(t)=0, L(t ; v)$ is a continuous function of $t$; moreover, if $\tilde{B}_{1}(t)=0, L(t ; v)=\tilde{B}_{2}(t)=(\phi-1) t^{\phi-1}>0$ unless $t=0$ in which case it equals 0 . 
Subcase (b.2): $v^{*}<v$ and $p \lambda \geq 1$. Now

$$
L(t ; v) \begin{cases}>0 & \text { for } 0<t<\xi_{1}, \\ <0 & \text { for } \xi_{1}<t<\xi_{2}, \\ >0 & \text { for } \xi_{2}<t,\end{cases}
$$

where

$$
\xi_{1}=\inf \left\{t>s_{2}: V(t)<v\right\} \quad \text { and } \quad \xi_{2}=\inf \left\{t>\xi_{1}: V(t)>v\right\} .
$$

Since $\eta(t)$ has two sign changes from + to - to + , according to Proposition 3.2, $r(t)$ must be either IFR, BT, or MBT. Using Proposition 3.1 again, we know that $r(t)$ is initially strictly increasing since $r^{\prime}(0+)=+\infty$ and BT is thus ruled out. Also, $r(0+)=p \lambda$ and, by assumption, $p \lambda \geq 1$, and so IFR is ruled out since $r(t)$ is ultimately strictly increasing to 1 as $t$ goes to $+\infty$, i.e. $r(t)$ must be MBT.

Subcase (b.3): $v^{*}<v$ and $p \lambda<1$. Using the analysis of subcase (b.2), we can no longer rule out IFR since $p \lambda<1$, Thus, $r(t)$ can be either IFR or MBT. Graphs of $r(t)$ indicate that both types are possible.

Case (c): $\lambda>1$ and $\phi=2$. For this choice of parameters, we obtain $s_{1}<t_{1}<0=$ $t_{2}=t_{3}=s_{2}$. Thus, $\tilde{B}_{1}(t)<0$ and $R(t)<0$ for all $t>0$. This implies that $V(t)$ is strictly increasing for all $t>0$ with

$$
V(0+)=\lim _{t \downarrow 0}\left\{\frac{\lambda \mathrm{e}^{t(1-\lambda)}\left[2(1-\lambda) t-(1-\lambda)^{2} t^{2}\right]-t}{\lambda \mathrm{e}^{t(1-\lambda)}\left[2(1-\lambda) t-(1-\lambda)^{2} t^{2}\right]}\right\}=1+\frac{1}{2 \lambda(\lambda-1)} .
$$

Again, we need to analyze separate subcases.

Subcase $(c .1): 1<v \leq 1+[2 \lambda(\lambda-1)]^{-1}$. Then $V(t)>v$ for all $t>0$ and so $L(t ; v)>0$, $t>0$. Consequently, $\eta(t)$ is strictly increasing, i.e. $r(t)$ is IFR.

Subcase (c.2): $1+[2 \lambda(\lambda-1)]^{-1}<v$. In this case,

$$
L(t ; v) \begin{cases}<0 & \text { for } 0<t<\xi \\ >0 & \text { for } \xi<t\end{cases}
$$

where

$$
\xi=\inf \{t>0: V(t)>v\} .
$$

So $\eta(t)$ now has one sign change from - to + . According to Proposition 3.2, $r(t)$ is either BT or IFR. From Proposition 3.1, $r^{\prime}(0+)=q\left(1-\lambda^{2} p\right)$. So, if $\lambda^{2} p<1, r^{\prime}(0+)>0$, which implies that $r(t)$ is IFR, while if $\lambda^{2} p>1, r^{\prime}(0+)<0$, which implies that $r(t)$ is BT. For the case $\lambda^{2} p=1, r^{\prime}(0+)=0$ does not distinguish between the two choices. However, we can use the analogous technique as in Block et al. (2003a) to calculate

$$
r^{\prime \prime}(0+)=\frac{(\lambda-1)^{3}(1+\lambda)}{\lambda^{3}}>0,
$$

and, hence, $r^{\prime}(t)>0$ in a neighborhood of $t=0$. Thus, again, $r(t)$ is IFR. See also Block $e t$ al. (2008, Section 4).

Case $(d): 0<\lambda<1$ and $\phi>2$. Here $t_{2}$ and $t_{3}$ are complex with $0<s_{2}<t_{1}<s_{1}$. Since $\tilde{B}_{1}(0)<0$, it follows that

$$
\tilde{B}_{1}(t) \begin{cases}<0, & 0<t<s_{2}, \\ >0, & s_{2}<t<s_{1}, \\ <0, & s_{1}<t\end{cases}
$$


while

$$
R(t) \begin{cases}<0, & 0<t<t_{1}, \\ >0, & t_{1}<t .\end{cases}
$$

So $V(t)$ is strictly increasing on $\left(0, s_{2}\right)$, strictly increasing on $\left(s_{2}, t_{1}\right)$, and strictly decreasing on $\left(t_{1}, \infty\right)$. We also have $V(0+)=1$ and $V(+\infty)=1$. Thus, it must be that $V\left(s_{2}-\right)=+\infty$, $V\left(s_{2}+\right)=-\infty, V\left(s_{1}-\right)=-\infty$, and $V\left(s_{1}+\right)=+\infty$ with $V(t)$ achieving a local maximum at $t=t_{1}$. If we calculate $V(t)$ at $t_{1}$, we obtain

$$
V\left(t_{1}\right)=1-\frac{\mathrm{e}^{1-\phi}(\phi-1)^{\phi-1}}{\lambda(1-\lambda)^{\phi-1} \Gamma(\phi)}<1 .
$$

(Actually, graphs show that $V\left(t_{1}\right)<0$ for all $0<\lambda<1$ and $\phi>2$.) Consequently, for $v>1$,

$$
V(t) \begin{cases}<v & \text { for } 0<t<\xi_{1}, \\ >v & \text { for } \xi_{1}<t<s_{2}, \\ <v & \text { for } s_{2}<t<s_{1}, \\ >v & \text { for } s_{1}<t<\xi_{2}, \\ <v & \text { for } \xi_{2}<t\end{cases}
$$

where

$$
\xi_{1}=\inf \left\{t<s_{2}: V(t)>v\right\} \quad \text { and } \quad \xi_{2}=\inf \left\{s_{1}<t: V(t)<v\right\} .
$$

Hence, from (A.2),

$$
L(t ; v) \begin{cases}<0, & 0<t<\xi_{1}, \\ >0, & \xi_{1}<t<\xi_{2}, \\ <0, & \xi_{2}<t,\end{cases}
$$

i.e. $\eta^{\prime}(t)$ has two sign changes from - to + to - . According to Proposition 3.2, $r(t)$ can be DFR, UB, or MUB.

From Proposition 3.1 we conclude that $r(t)$ cannot be DFR since $r(+\infty)=\lambda>p \lambda=$ $r(0+)$. Also, since $r^{\prime}(0+)=-p q \lambda^{2}<0$, it cannot be UB. Hence it must be MUB.

Case (e): $0<\lambda<1$ and $1<\phi<2$. In this case, $t_{3}<s_{2}<0<t_{2}<t_{1}<s_{1}$. Using the facts that $\tilde{B}_{1}(0)>0$ and $R(0)>0$, we have

$$
\tilde{B}_{1}(t) \begin{cases}>0, & 0<t<s_{1}, \\ <0, & s_{1}<t\end{cases}
$$

and

$$
R(t) \begin{cases}>0, & 0<t<t_{2}, \\ <0, & t_{2}<t<t_{1}, \\ >0, & t_{1}<t .\end{cases}
$$

Consequently, $V(t)$ is strictly decreasing on $\left(0, t_{2}\right)$, strictly increasing on $\left(t_{2}, t_{1}\right)$, and then strictly decreasing on $\left(t_{1}, s_{1}\right)$ and on $\left(s_{1}, \infty\right)$. Since $V(0+)=1$ and $V(+\infty)=1$, it follows that $V\left(s_{1}-\right)=-\infty$ and $V\left(s_{1}+\right)=+\infty$ with $V\left(t_{1}\right)$ a local maximum. The expression for $V\left(t_{1}\right)$ is exactly as in case (d) and so $V\left(t_{1}\right)<1$. Thus, for fixed $v>1$,

$$
V(t) \begin{cases}<v & \text { if } 0<t<s_{1}, \\ >v & \text { if } s_{1}<t<\xi, \\ <v & \text { if } \xi<t\end{cases}
$$


where

$$
\xi=\inf \left\{t>s_{1}: V(t)<v\right\}
$$

Hence, we conclude that

$$
L(t ; v) \begin{cases}>0 & \text { if } 0<t<s_{1}, \\ >0 & \text { if } s_{1}<t<\xi, \\ <0 & \text { if } \xi<t,\end{cases}
$$

and so $\eta^{\prime}(t)$ has only one sign change which goes from + to - . Thus, by Proposition 3.2, $r(t)$ is either DFR or UB. But, from Proposition 3.1, $r^{\prime}(0+)=+\infty$; it must therefore be UB.

Case $(f): 0<\lambda<1$ and $\phi=2$. For this, we obtain $0=s_{2}=t_{2}=t_{3}<t_{1}<s_{1}$. It then follows that

$$
\tilde{B}_{1}(t) \begin{cases}>0 & \text { for } 0<t<s_{1}, \\ <0 & \text { for } s_{1}<t,\end{cases}
$$

since $\tilde{B}_{1}(+\infty)=-\infty$, and

$$
R(t) \begin{cases}<0 & \text { for } 0<t<t_{1}, \\ >0 & \text { for } t_{1}<t,\end{cases}
$$

since $R(+\infty)=+\infty$. Consequently, $V(t)$ is strictly increasing on $\left(0, t_{1}\right)$, and strictly decreasing on $\left(t_{1}, s_{1}\right)$ and on $\left(s_{1}, \infty\right)$. Also,

$$
V(0+)=1-\frac{1}{2 \lambda(1-\lambda)}<0
$$

for $0<\lambda<1, V\left(s_{1}-\right)=-\infty, V\left(s_{1}+\right)=+\infty, V(+\infty)=1$, and $V(t)$ has a local maximum at $t=t_{1}$. From (A.4) with $\phi=2$,

$$
V\left(t_{1}\right)=1-\frac{1}{\lambda(1-\lambda) \mathrm{e}}<0 .
$$

Consequently, for fixed $v>1$,

$$
V(t) \begin{cases}<v & \text { for } 0<t<s_{1} \\ >v & \text { for } s_{1}<t<\xi \\ <v & \text { for } \xi<t\end{cases}
$$

where

$$
\xi=\inf \left\{t>s_{1}: V(t)<v\right\}
$$

and so

$$
L(t ; v) \begin{cases}>0 & \text { if } 0<t<s_{1}, \\ >0 & \text { if } s_{1}<t<\xi \\ <0 & \text { if } \xi<t\end{cases}
$$

Thus, $\eta^{\prime}(t)$ has only one sign change which goes from + to - . As before (see Proposition 3.2), we conclude that $r(t)$ is either DFR or UB. Proposition 3.1 gives $r^{\prime}(0+)>0$, and, hence, $r(t)$ is UB.

Case $(g): \lambda=1$ and $1<\phi$. This case was considered in Glaser (1980). 


\section{References}

Block, H. And Joe, H. (1997). Tail behavior of the failure rate functions of mixtures. Lifetime Data Anal. 3, 269-288.

Block, H. W., LANGBERG, N. A. AND SAvits, T. H. (2012). A mixture of exponentials and IFR gamma distributions having an upsidedown bathtub-shaped failure rate. Prob. Eng. Inf. Sci. 26, 573-580.

Block, H. W., LI, Y. AND SAvits, T. H. (2003a). Initial and final behaviour of failure rate functions for mixtures and systems. J. Appl. Prob. 40, 721-740.

Block, H. W., Mi, J. and Savits, T. H. (1993). Burn-in and mixed populations. J. Appl. Prob. 30, 692-702.

Block, H. W., Savits, T. H. and Wondmagegnehu, E. T. (2003b). Mixtures of distributions with increasing linear failure rates. J. Appl. Prob. 40, 485-504.

Block, H. W., Langberg, N. A., Savits, T. H. and Wang, J. (2010). Continuous mixtures of exponentials and IFR gammas having bathtub-shaped failure rates. J. Appl. Prob. 47, 899-907.

Block, H. W., LI, Y., SAVITs, T. H. AND WANG, J. (2008). Continuous mixtures with bathtub-shaped failure rates. J. Appl. Prob. 45, 260-270.

Esary, J. D., Marshall, A. W. and Proschan, F. (1970). Some reliability applications of the hazard transform. SIAM J. Appl. 18, 849-860.

Glaser, R. E. (1980). Bathtub and related failure rate characterizations. J. Amer. Statist. Assoc. 75, 67-672.

Gupta, R. C. AND Warren, R. (2001). Determination of change points of non-monotonic failure rates. Commun. Statist. Theory Meth. 30, 1903-1920.

Kemperman, J. H. B. (1991). Mixtures with a limited number of modal intervals. Ann. Statist. 19, 2120-2144.

LAI, C.-D. AND XIE, M. (2006). Stochastic Ageing and Dependence for Reliability. Springer, New York.

Marshall, A. W. ANd Olkin, I. (2007). Life Distributions. Springer, New York.

Savits, T. H. (2003). Preservation of generalized bathtub-shaped functions. J. Appl. Prob. 40, 473-484. 